Cipango $\begin{aligned} & \text { Cipango } \\ & \text { Cahiers d'études japonaises }\end{aligned}$

18 | 2011

Le Japon et le fait colonial I

\title{
Un autre regard : la Mandchourie des photographes pictorialistes japonais
}

Sandrine Dalban-Tabard

\section{OpenEdition}

1 Journals

Édition électronique

URL : https://journals.openedition.org/cipango/1522

DOI : $10.4000 /$ cipango. 1522

ISSN : 2260-7706

Éditeur

INALCO

Édition imprimée

Date de publication : 1 juin 2011

Pagination : 79-104

ISBN : 978-2-85831-195-8

ISSN : $1164-5857$

Référence électronique

Sandrine Dalban-Tabard, « Un autre regard : la Mandchourie des photographes pictorialistes

japonais », Cipango [En ligne], 18 | 2011, mis en ligne le 14 juin 2013, consulté le 30 juin 2021. URL:

http://journals.openedition.org/cipango/1522 ; DOI : https://doi.org/10.4000/cipango.1522

Ce document a été généré automatiquement le 30 juin 2021.

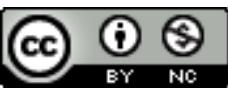

Cipango est mis à disposition selon les termes de la Licence Creative Commons Attribution - Pas d'Utilisation Commerciale 4.0 International. 


\title{
Un autre regard : la Mandchourie des photographes pictorialistes japonais
}

\author{
Sandrine Dalban-Tabard
}

1 L'anthropologue Mariko Tamanoi a publié en 2009 un ouvrage intitulé Memory Maps: The State and Manchuria in Postwar Japan, dans lequel l'auteur revient sur l'histoire de la Mandchourie telle qu'elle a été vécue par des Japonais et des Chinois dont elle a recueilli les témoignages. Au-delà de l'intérêt de cet ouvrage, c'est la couverture qui a d'abord retenu notre attention. On y voit une photographie de 1934, prise par Terashima Manji - ou Banji - 寺嶋萬治 (1898-1983), de type pictorialiste, montrant des moutons sous un arbre au milieu d'un paysage qui s'étend au loin (fig. 1). En préambule, Tamanoi explique comment cette photographie a influé sur le destin de Memory Maps. Cette représentation romantique de la Mandchourie qui correspondait si bien, écritelle, à l'image qu'elle se faisait de cette ancienne colonie japonaise, attira en effet son attention: «Il était aisé pour qui regardait cette photographie de succomber à la nostalgie pour le passé impérial du Japon. Ainsi, en voyant cette photographie, je ne pus m'empêcher de ressentir de la nostalgie pour cette terre dont j'avais tant entendu parler pendant mon enfance au Japon $»^{1}$. À la fin du processus d'écriture, Mariko Tamanoi contacta le détenteur des droits de la photographie, un ancien élève de Terashima. Celui-ci lui expliqua alors que le site représenté sur la photographie était connu à l'époque comme le lieu d'exécutions de rebelles chinois par l'armée japonaise. Ces informations amenèrent Tamanoi à reconsidérer son propos et à réécrire son étude en prenant en compte le poids des représentations; elle décida néanmoins de conserver la photographie de Terashima en couverture.

2 La genèse de Memory Maps peut paraître anecdotique. Elle pose néanmoins la question du rôle joué par le pictorialisme dans la mémoire japonaise : comment ce style de photographie amateur à visée artistique a-t-il participé à la formation d'une certaine image de la Mandchourie? 
Figure 1

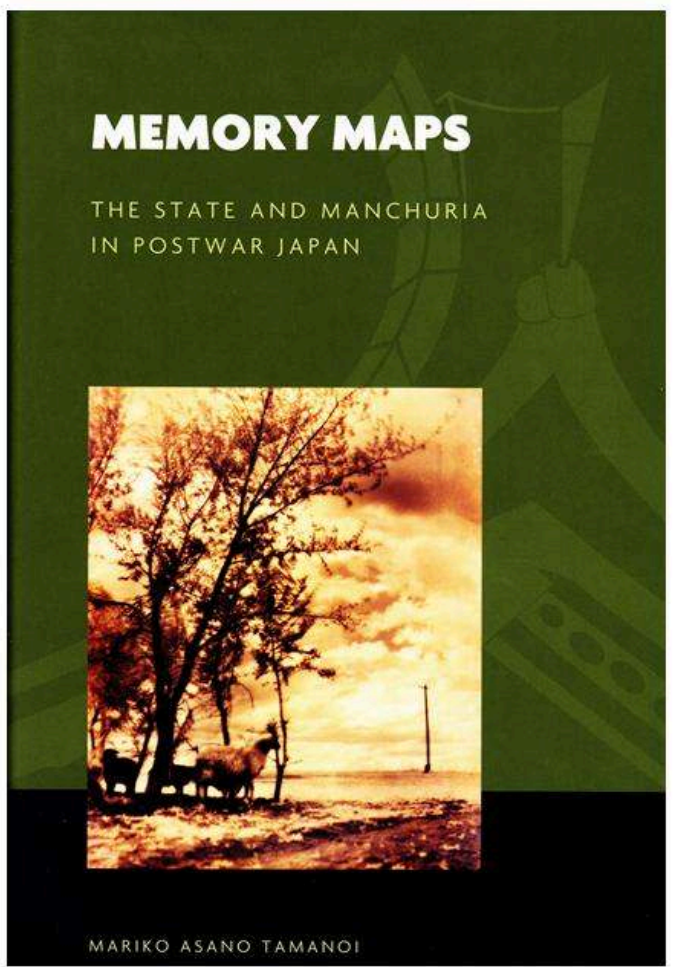

Couverture de Memory Maps de Mariko Tamanoi, Univ. of Hawai'i Press, 2009

\section{Le pictorialisme, photographie amateur à visée artistique}

Que désigne-t-on sous le nom de "pictorialisme $»^{2}$ ? Il s'agit d'une pratique artistique de la photographie, faite en amateur, qui s'est développée des années 1880 à 1920 en Europe $^{3}$ et aux États-Unis ${ }^{4}$, et des décennies 1890 à 1930 au Japon $^{5}$. Les photographes pictorialistes procédaient à des manipulations de l'image photographique (retouches, déformations ou encore montages), qui avaient pour but d'éloigner la photographie à la fois de sa nature documentaire, instantanée, et de son application commerciale (photographie de studio). Le principe esthétique sur lequel reposait cette approche de la photographie était l'expression des sentiments du photographe. Le pictorialisme se définit également par les techniques employées. En Europe et en Amérique du Nord comme au Japon, deux courants ont évolué parallèlement: ceux refusant la manipulation de l'image et jouant prioritairement sur la lumière et la composition, et ceux pratiquant la retouche et travaillant leurs épreuves comme des toiles grâce à l'usage de gomme bichromate ou arabique, ou encore de la colle 6 .

4 L'étude de la participation, directe et indirecte, des photographes pictorialistes à l'évolution des représentations de la société japonaise d'avant-guerre a, jusqu'à présent, été négligée. La nature amateur et artistique de cet usage de la photographie, tout autant que l'aspect atemporel de nombres d'images, ont pu laisser penser que ces photographes ne s'intéressaient pas à leurs contemporains. En outre, dans l'histoire de la photographie japonaise, ce sont les formes modernistes de photographie, telles que 
le photomontage et le photoreportage, qui sont présentées comme représentatives des années 1930 et du nouveau regard porté par les photographes, amateurs comme professionnels, sur la société.

Figure 5

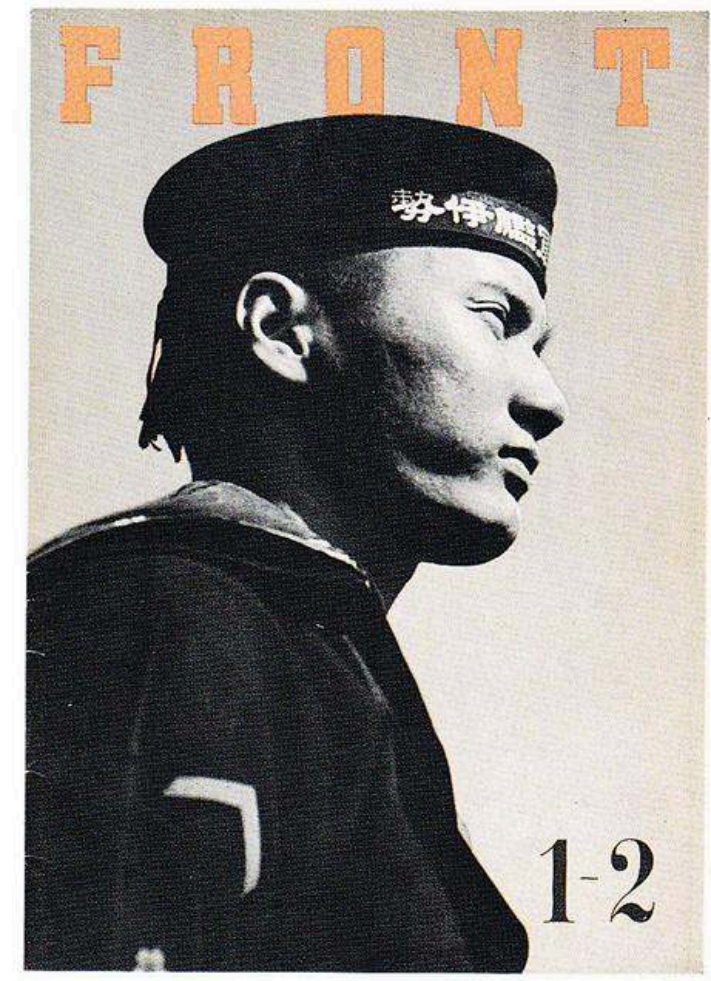

COUVERTURE DU NUMÉRO 1-2, DE FRONT, 1942.

Les années 1930 furent effectivement marquées par le développement de la photographie moderniste, utilisée dans un premier temps à des fins commerciales (débuts de la photographie publicitaire) ; elle fut ensuite employée à des fins politiques, comme le souligne le rôle joué par les revues Nippon ou Front $^{7}$ (fig. 5) dans la communication du Japon auprès des grandes puissances de l'époque et l'importance des photographies murales (shashin hekiga 写真壁画 ou foto-myūraru フォト・ミューラル) présentées à l'Exposition universelle de 1939, à New York ${ }^{8}$. Dans le domaine de la photographie commerciale, des albums photographiques présentant des localités taïwanaises ou coréennes furent mis sur le marché dès les années 1910 ; puis l'offre fut élargie aux albums présentant les îles indonésiennes, la Mandchourie (puis le Mandchoukouo). L'évocation de Taiwan s'est répandue dans le Japon des années 1920 avec la fabrication de fonds peints sur tissu vendus aux photographes professionnels (fig. 6), constituant l'arrière-plan de portraits individuels ou de famille. Avec le développement $\mathrm{du}$ tourisme continental apparut une mode des albums photographiques réalisés en souvenir d'un voyage de groupe dans les territoires japonais 9 . 
Figure 6

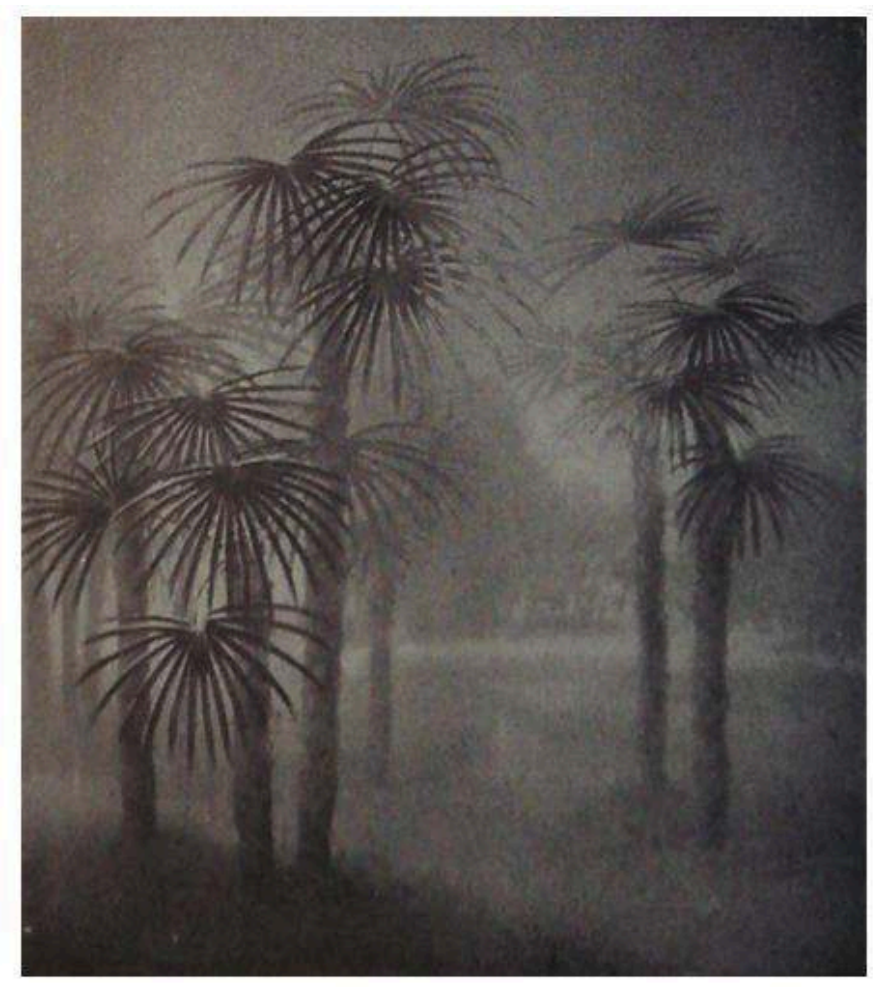

EXEMPLe d'ARRIÈre-PLAN EN VENTE En 1929. CATAlogue ASANUMA, 1929, N. P.

6 Pourtant, les représentations des territoires des colonies et territoires sous mandat n'étaient pas cantonnées à ces secteurs : à la fin des années 1920, paysages et portraits pictorialistes furent diffusés dans des revues destinées aux photographes amateurs. Ces photographies montrent l'intérêt porté par de nombreux amateurs pour ces territoires et remettent en cause l'idée d'un pictorialisme par nature étranger au fait contemporain. Nous discuterons donc ici le rôle joué par le pictorialisme dans la production et la diffusion de représentations de la Mandchourie au travers des liens de certains photographes avec la Compagnie sud-mandchourienne de chemin de fer, ou Mantetsu 満鉄 ${ }^{10}$, et nous dégagerons, dans un second temps, les particularités de cette iconographie.

\section{Photographes pionniers ou pionniers photographes?}

7 D'après les annuaires publiés dans différentes éditions de la Revue annuelle de photographie (Nihon shashin nenkan 日本写真年鑑) ${ }^{11}$, le nombre de photographes inscrits dans un club de Mandchourie était passé de quatre-vingt-douze en 1926, à quatre cent quatre-vingt-neuf en 1930. Le nombre de studios, quant à lui, doubla entre 1926 et 1930 (passant de dix-sept à trente-sept). Parmi ceux-ci, trois photographes en particulier sont connus pour leur travail en Mandchourie à partir des années 1920. Il s'agit de Terashima Manji cité par Mariko Tamanoi, Fuchikami (Fuchigami) Hakuyō 淵上白陽 (1889-1960) et Sakakibara Shōichi 榊原正一 (1897-1980).

8 Terashima quitta l'archipel pour le continent en 1923 ; il tint un studio photographique, le Terashima shashinkan 寺嶋写真館, dans la ville de Moukden ${ }^{12}$ jusqu'en 1929, année 
où il quitta la Mandchourie pour Los Angeles. Il revint en Mandchourie en 1933 et devint, en 1940, le président de l'Association des photographes de Mandchourie (Zen Manshū shashinka kyōkai 全満州写真家協会).

Fuchikami, quant à lui, quitta Kōbe en 1927, où il avait créé la revue Hakuyō consacrée au pictorialisme, pour entrer au service de la Compagnie sud-mandchourienne de chemin de fer où il poursuivit son œuvre de photographe jusqu'en 1941, date à laquelle il démissionna et rentra en métropole.

Enfin, Sakakibara Shōichi, photographe pictorialiste proche de Fuchikami Hakuyō, partit pour la Mandchourie en 1910 et entra au service de la Mantetsu où il occupa différents postes à responsabilité ; il fonda également en 1931 à Dairen (Dalian) une association de photographes appelée le Club de la nouvelle lumière (Shinkō kurabu 新 光俱楽部) et l'année suivante l'Association des photographes de Mandchourie (Manshū shashin sakka kyōkai 満州写真作家協会) ${ }^{13}$.

11 De ces trois photographes, Fuchikami fut celui qui œuvra le plus activement à la création et à la diffusion d'une image pictorialiste de la Mandchourie. En 1930, il écrivait dans la préface de Manshū shashin nenkan 満州写真年鑑 ${ }^{14}$, première revue photographique annuelle de Mandchourie, dont il dirigeait la publication ${ }^{15}$ :

C'est la première fois qu'est publiée une revue annuelle dans le domaine de la photographie en Mandchourie. Je voudrais que les chefs d'œuvres réalisés par un petit nombre de photographes soient regardés non pas seulement par la scène photographique japonaise, mais par les photographes du monde entier.

De plus, nous sommes les pionniers de la Mandchourie. Les œuvres réunies ici ne sont pas de simples œuvres artistiques; elles proclament cette idée importante.

満州の写壇から年間を出版するのはこれが始めてである。小数な写真家によっ て、これだけの绦れた作品が生み出されたことを、日本だけでなく世界の写壇 から観て貴いたい。そして満州の写真家を譛めて欲しい。

それに吾々は満州の開拓者である。年鑑に蒐められた作品にも、単に芸術品と してばかりでなしに、その重大な意義が譫はせてある。

Cet extrait nous éclaire sur les ambitions portées par Fuchikami pour la photographie qu'il pratiquait. Il souhaitait à la fois porter à la connaissance d'un public dépassant les frontières du Japon les qualités des photographes japonais et se placer, lui et les photographes ayant participé à cette revue, comme "pionniers de la Mandchourie " ("Wareware wa Manshū no kaitakusha de aru»), inscrivant ainsi la photographie artistique dans le projet de développement industriel et agricole de la région, et sousentendant que les photographes de Mandchourie se situaient à l'avant-garde des photographes japonais. Fuchikami se percevait donc, ainsi que ses confrères, à la fois comme des pionniers de la photographie et des pionniers en Mandchourie. L'engagement de Fuchikami est connu; il était en charge de la communication de la Mantetsu, ce qui incluait notamment la publication de la revue Kyōwa 協和 (Concorde) à partir de 1928 et de Manshū gurafu 満州グラフ (L'Illustré de Mandchourie) en $1933^{16}$.

Il existe d'autres exemples de relations entre photographes pictorialistes et Mantetsu. En 1929, sept photographes, dont Akiyama Tetsusuke 秋山敨輔 (1880-1944) ${ }^{17}$, Yamamoto Makihiko山本牧彦 (1893-1985) ${ }^{18}$, Sakakibara Seiyō 榊原青葉 $(1887-1974)^{19}$ d'éminents membres du monde pictorialiste - avaient été invités par la Mantetsu à participer à une excursion photographique de treize jours les ayant conduits à Dairen, Moukden, Ryojun (Lüshun) ou encore Harbin. Quatre-vingt-quatorze de leurs photographies furent sélectionnées pour être exposées dans l'archipel au sein de l'Exposition artistique de photographies sur la Mandchourie (Manshū shashin bijutsu- 
ten 満州写真美術展) organisée en 1930 par la Mantetsu. Par ailleurs, suite à la création du Mandchoukouo en 1932, l'État japonais fit appel à des photographes pictorialistes, dont Terashima Manji, rassemblés dans le club amateur formé au sein de la Mantetsu pour promouvoir l'image du nouvel État. Les œuvres de ces photographes furent présentées à l'Exposition universelle de Chicago en 1933, dans le pavillon de la Mantetsu, et ce fut un tel succès que cette exposition de photographies pictorialistes fut présentée dans tous les États-Unis. Terashima et ce groupe de pictorialistes, à leur retour au Mandchoukouo, travaillèrent activement à la promotion de la Mantetsu et de sa politique de développement du territoire ${ }^{20}$.

Les parcours de Fuchikami et Terashima illustrent les liens unissant au début des années 1930 certains pictorialistes et pouvoir politique, et soulignent les ambitions portées par de nombreux photographes installés en Mandchourie pour leur art, mais aussi pour leur pays. En ce sens, il est possible d'évoquer un "pictorialisme engagé " comme revendiqué par Fuchikami Hakuyō dans sa préface à Manshū shashin nenkan, et conçu pour s'intégrer à la propagande mise en place autour de l'expansion japonaise, notamment dans la revue Mansh ü Graph - ce qui contredit l'idée communément admise selon laquelle ce genre photographique s'inscrivait dans le refus du présent.

\section{Iconographie pictorialiste de la Mandchourie}

La question de la perception photographique de la Mandchourie ne se limite pas à celle de l'engagement de certains photographes et de leur volonté de participer à un projet dépassant le monde de la photographie. Les œuvres de type pictorialistes publiées dans les revues de photographie amateur vers 1930 nous renseignent sur la façon dont était alors perçu ce territoire d'outre-mer et révèle la construction d'une image duale : d'une part, les photographies représentant des activités industrielles et la mécanisation; de l'autre, les photographies de paysages et de types.

16 Les photographies pictorialistes illustrant la vie en Mandchourie représentaient une faible part des œuvres publiées dans des revues pour amateurs comme Nihon shashin nenkan. Ainsi, l'édition de 1928 ne présente-t-elle aucun exemple de ce type de photographies; dans celle de 1929, cinq photographies sur les cent huit reproduites se rapportent à la Mandchourie. Ce ratio est de neuf pour cent neuf dans l'édition de 1930, de cinq sur cent dix en 1931, de cinq sur quatre-vingt-huit en $1933^{21}$. La publication d'œuvres dans les revues de photographie était soumise à l'appréciation d'un jury, généralement limité aux rédacteurs. Ni les choix du jury ni les intentions des auteurs n'étaient commentés, ce qui ne permet pas de trancher de manière définitive la question de la représentativité quantitative de ces œuvres dans le corpus général. La comparaison du nombre de photographes amateurs et professionnels recensés dans l'annuaire de Nihon shashin nenkan ${ }^{22}$ au nombre de ceux installés en Mandchourie, montre les proportions suivantes: en 1928, 0,36\% des photographes recensés sont domiciliés en Mandchourie, en 1929, ce nombre monte à 1,18 \%, en 1930, à $2,25 \%$ et en 1931 il redescend à $1,43 \%$. Le nombre de photographes japonais installés en Mandchourie était donc faible; néanmoins leurs œuvres étaient publiées dans des proportions plus importantes. Nous ignorons la proportion de photographies sélectionnées par rapport au nombre de celles traitant de ce thème soumises à la rédaction des revues. Les photographes de Mandchourie étaient-ils plus productifs que ceux de l'archipel? Leur travail était-il de meilleure qualité? Les images de 
Mandchourie étaient-elles prisées des rédacteurs ou des lecteurs, et à ce titre leur publication était-elle favorisée? Ou bien n'était-ce que certaines œuvres, fruit du travail de photographes ayant déjà acquis une certaine renommée, comme Fuchikami Hakuyō, ou de photographes faisant partie des relations des rédacteurs, auxquelles était donnée la priorité ? Voici autant de questions qui restent sans réponse pour le moment, principalement en raison du désintérêt qui les a entourées jusqu'à présent.

Figure 7

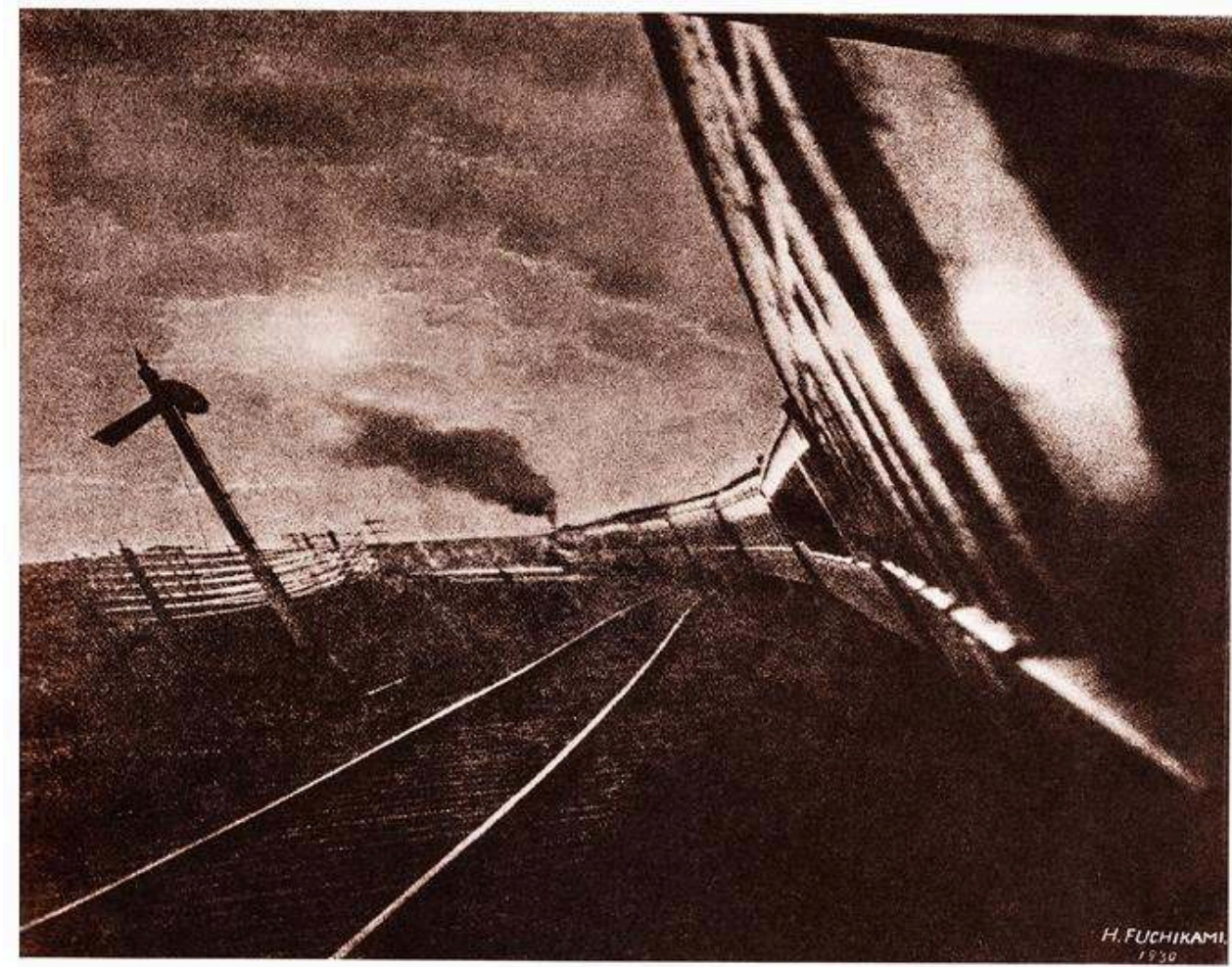

Fuchikami Hakuyō 淵上白陽, Ressha bakushin 列車驀進 (Train à pleine vitesse), Asahi Kamera, 1930.

Du point de vue thématique, il est intéressant de souligner que les images pictorialistes ayant pour thème l'activité industrielle étaient minoritaires dans les revues, même si ce sont celles auxquelles il est le plus fréquemment fait référence dans l'histoire de la photographie japonaise pour illustrer le travail des pictorialistes sur le continent ${ }^{23}$. Un des exemples les plus connus est une œuvre de Fuchikami Hakuyō, Ressha bakushin 列車 葍進 (Train à pleine vitesse), parue en 1930 dans la revue de photographie amateur Asahi kamera (fig. 7) et célébrant la puissance de la machine et les grands espaces. Les représentations du monde industriel ont en commun de proposer un cadrage serré, une part importante de l'espace étant occupée par l'usine ou l'activité représentées; l'atmosphère y semble oppressante et les noirs épais évoquent l'odeur lourde de la graisse des machines ou l'atmosphère chaude d'une fonderie (fig. 8). 
Figure 8

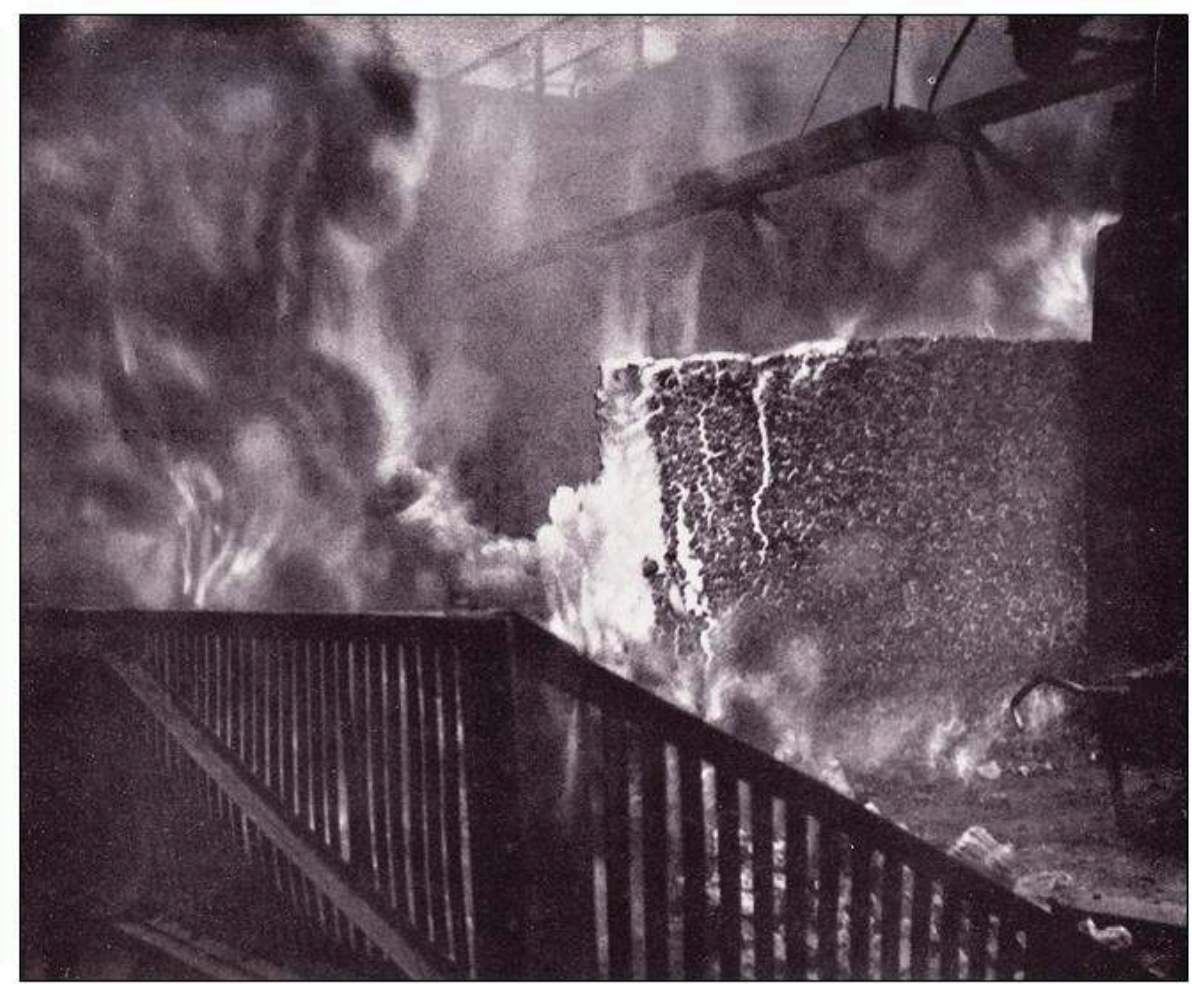

Fuchikami Hakuyō 淵上白陽, Netsu 熱 (Forte chaleur), Nihon shashin nenkan, 1933, p. 58.

18 La publication d'un nombre restreint de photographies pictorialistes sur ce thème peut paraître surprenante, notamment au regard de la présence un peu plus marquée des œuvres montrant des usines ou des travailleurs des grandes villes de l'archipel. En effet, la Mandchourie était présentée - et par certains, comme Fuchikami, perçue comme une terre pleine de promesses pour le futur industriel du Japon, un espace où réaliser l'utopie mécanique louée notamment par le constructivisme et le futurisme. Le faible nombre d'œuvres publiées sur ce thème peut tenir au fait qu'au début des années 1930 le gouvernement japonais avait émis de nombreuses recommandations concernant la non-publication d'informations touchant de près ou de loin au déploiement politique, économique et militaire du Japon en Mandchourie ${ }^{24}$. On peut y voir également l'expression de l'intérêt limité généralement exprimé par les pictorialistes pour les paysages industriels ${ }^{25}$ : les photographes arrivant en Mandchourie, même s'ils avaient accès à des équipements industriels et à leurs alentours, n'étaient peut-être pas attirés par cet aspect de la réalité mandchoue alors que les photographes modernistes, au contraire, ont beaucoup travaillé sur la représentation du monde industriel, sur l'archipel comme en Mandchourie. 
Figure 9

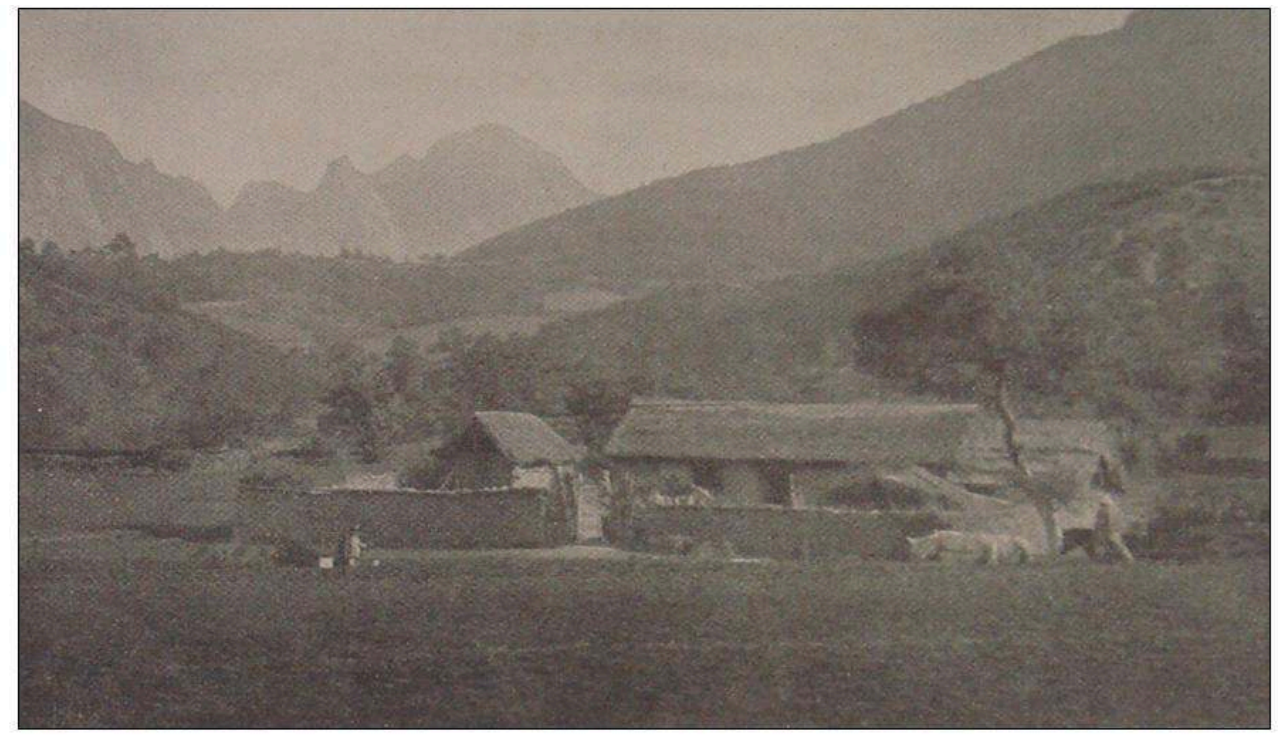

Tabata Eizō 田畑英三, Jōka 盛夏 (Plein été), Nihon shashin nenkan, 1929, p. 69.

En revanche, l'attention des photographes pictorialistes s'est plus volontiers portée sur les paysages et les portraits, et en particulier sur ceux incluant des aperçus du mode de vie des populations autochtones. Certaines de ces photographies de paysage ne sont pas différentes, dans leur forme, de paysages pictorialistes réalisés sur l'archipel, comme celle proposée par Tabata Eizō 田畑英二 ${ }^{26}$ en 1929 (fig. 9) : une ferme isolée au pied d'une chaîne de montagnes naissantes, la silhouette effacée d'un homme et deux chevaux. La forme des montagnes à l'arrière-plan évoque la Chine; sans cela et la légende, cette photographie aurait pu être prise dans l'archipel. Certains clichés renvoient plus explicitement à la Chine, comme cette épreuve de Nakane Shun.yō 中根 春洋 montrant une ferme étrangement déformée, devant laquelle se trouve un cochon (fig. 10), type de représentation dont on ne trouve aucune occurrence dans les images produites à l'époque dans l'archipel ${ }^{27}$; enfin, aucun doute n'est permis, l'image étant légendée Tetsurei shoken 鉄嶺所見 (Vue de Tieling ${ }^{28}$ ). Les images de la Mandchourie rurale comprennent également des représentations de travaux agricoles, l'accent étant souvent mis sur les animaux de trait (bœufs, ânes ${ }^{29}$, fig. 12). 
Figure 10

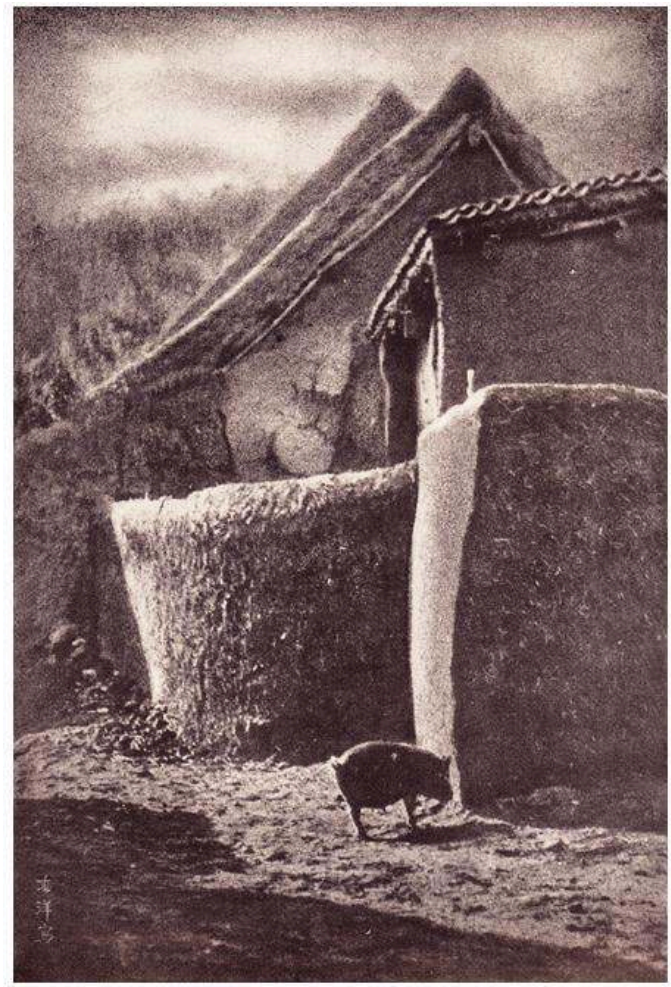

Nakane Shun.yō 中根春洋, Tetsurei shoken 鉄嶺所見 (Vue de Tienling), Nihon shashin nenkan, 1930, p. 67

\section{Figure 11}

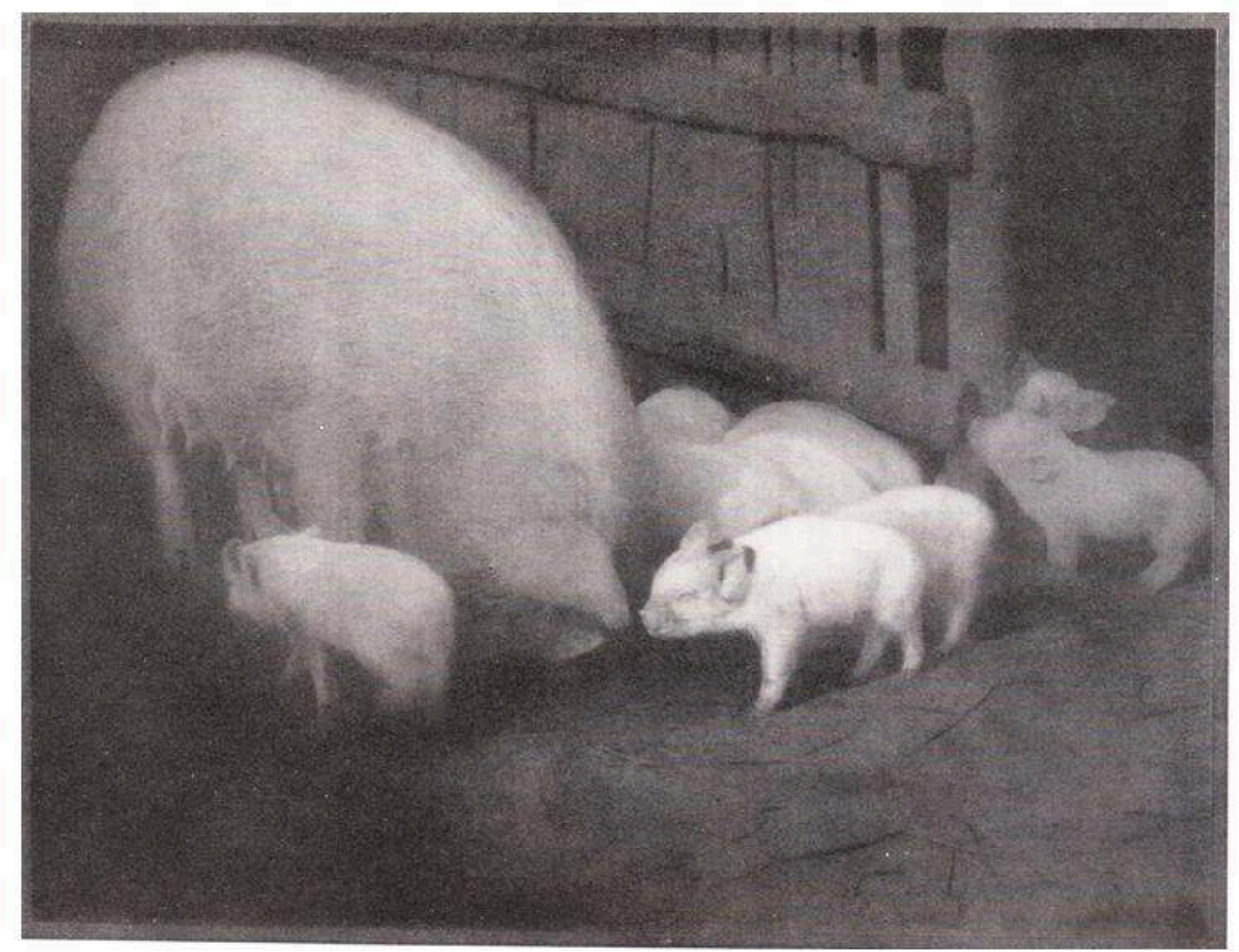

Takio Shakyō 瀧尾寫狂, Buta 豚 (Porcs), Nihon shashin nenkan, 1930, p. 15. 
Figure 12

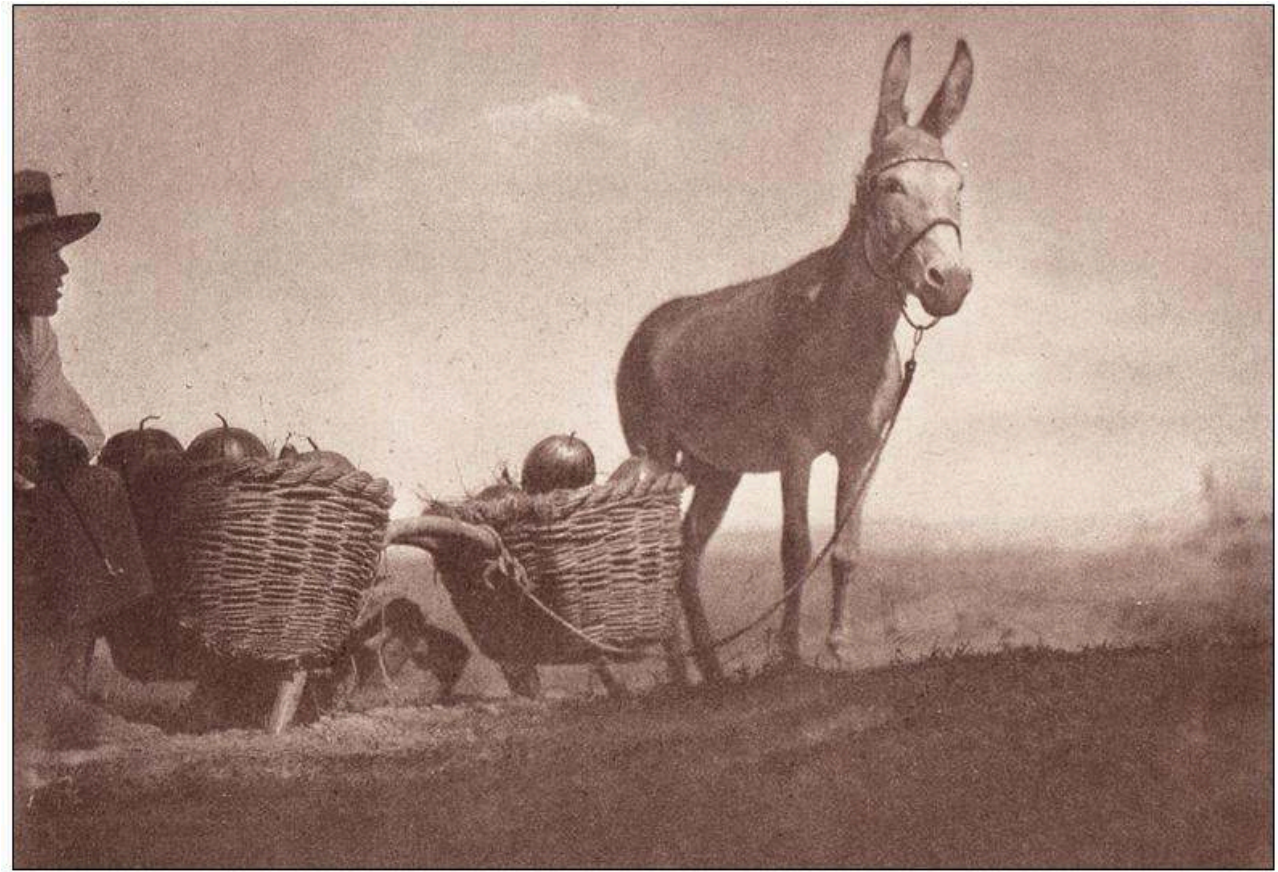

Tsuchida Kan.ichi 土田寛一, Roba 驢馬 (Âne), Nihon hashin nenkan, 1930, p. 5

Cependant, le motif le plus représenté consiste en des scènes de rue composées de groupes de personnages anonymes, souvent privés de visage. Ces images évoquent, notamment par des déformations, une atmosphère oppressante de désordre, absente de l'iconographie pictorialiste relative à la vie sur l'archipel (fig. 13). Certaines photographies évoquent le départ, montrant des Chinois dos à l'objectif, dans des décors inquiétants (fig. 14) qui ne sont pas sans rappeler l'univers expressionniste du Cabinet du docteur Caligari. Ces représentations de paysages comportent également des images étranges, comme le Paysage avec chameaux (Rakuda no iru fükei 駱鳥他のいる風景, fig. 15) de Kawamura Torakichi 川村虎吉, ou angoissantes, comme la photographie de Shiba Yōson 志波陽村 qui montre un homme dont le visage est partiellement caché par son chapeau et qui semble s'approcher du photographe - et du spectateur - de façon menaçante (fig. 16). Enfin, il faut mentionner les portraits, que l'on pourrait qualifier d'ethnographiques, et qui, dans leur répétition, semblent vouloir dresser un portrait de la population autre que japonaise présente en Mandchourie : enfants pauvres au regard vague (fig. 17) ou encore femme lavant le linge dans un baquet à même le sol d'une cour en terre battue, dans une lumière blanche et froide (fig. 18). 
Figure 13

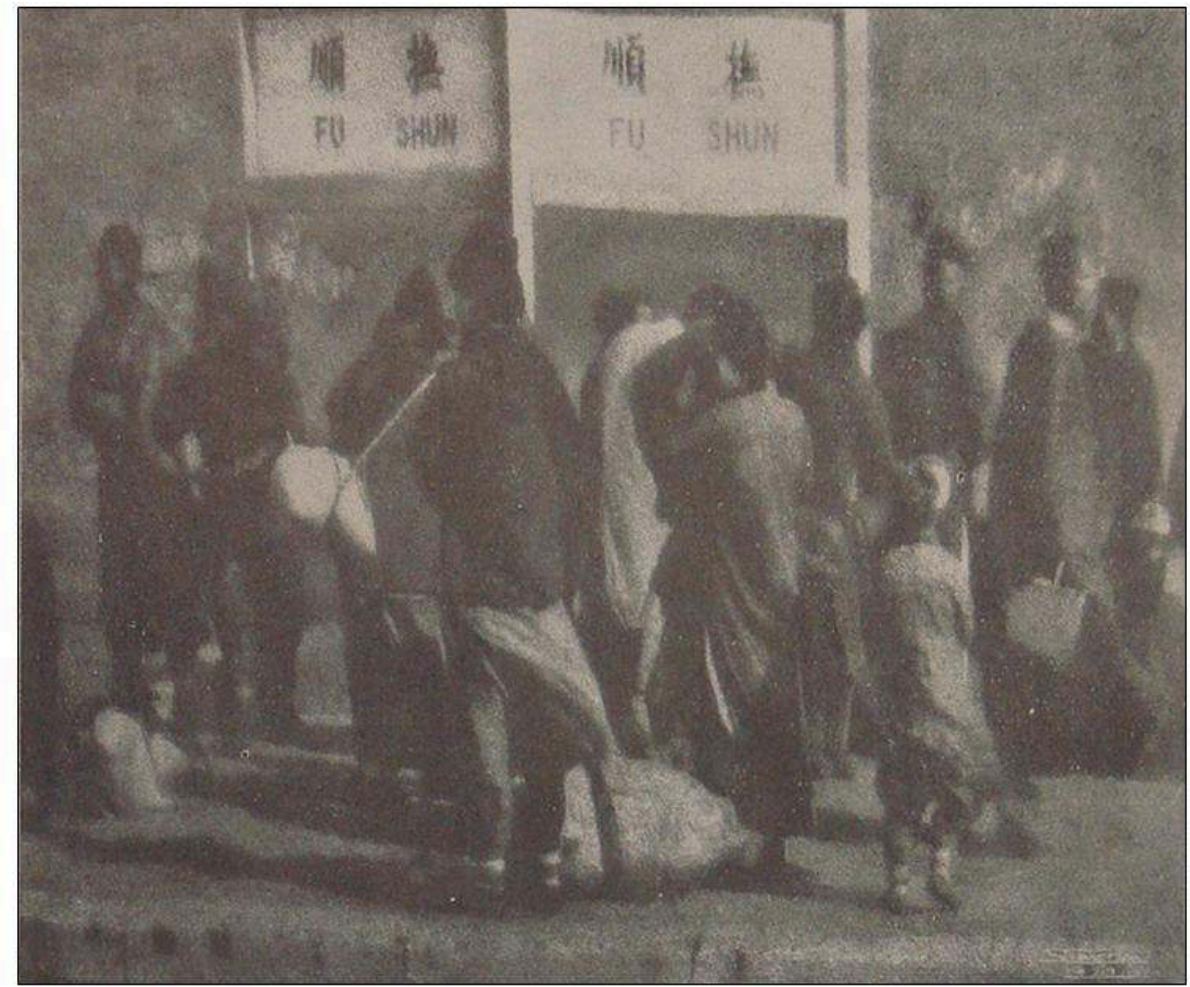

Umeda Shōtarō 梅田正太郎, Ekitō zakkan 駅頭雑観 (Vue de la gare), Nihon shashin nenkan, 1929, p. 71.

\section{Figure 14}

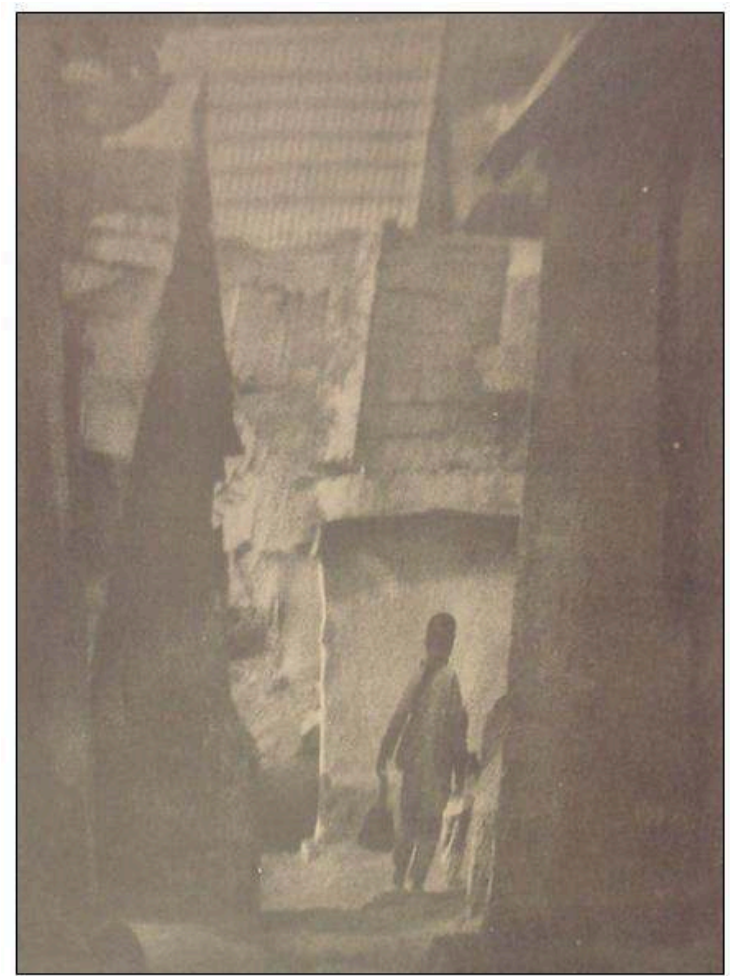

Fuchikami Hakuyō 淵上白陽, Ishi dōgai shōkei 石道街小景 (Rue pavée) Nihon shashin nenkan, 1929, p. $72 \mathrm{~A}$. 
Figure 15

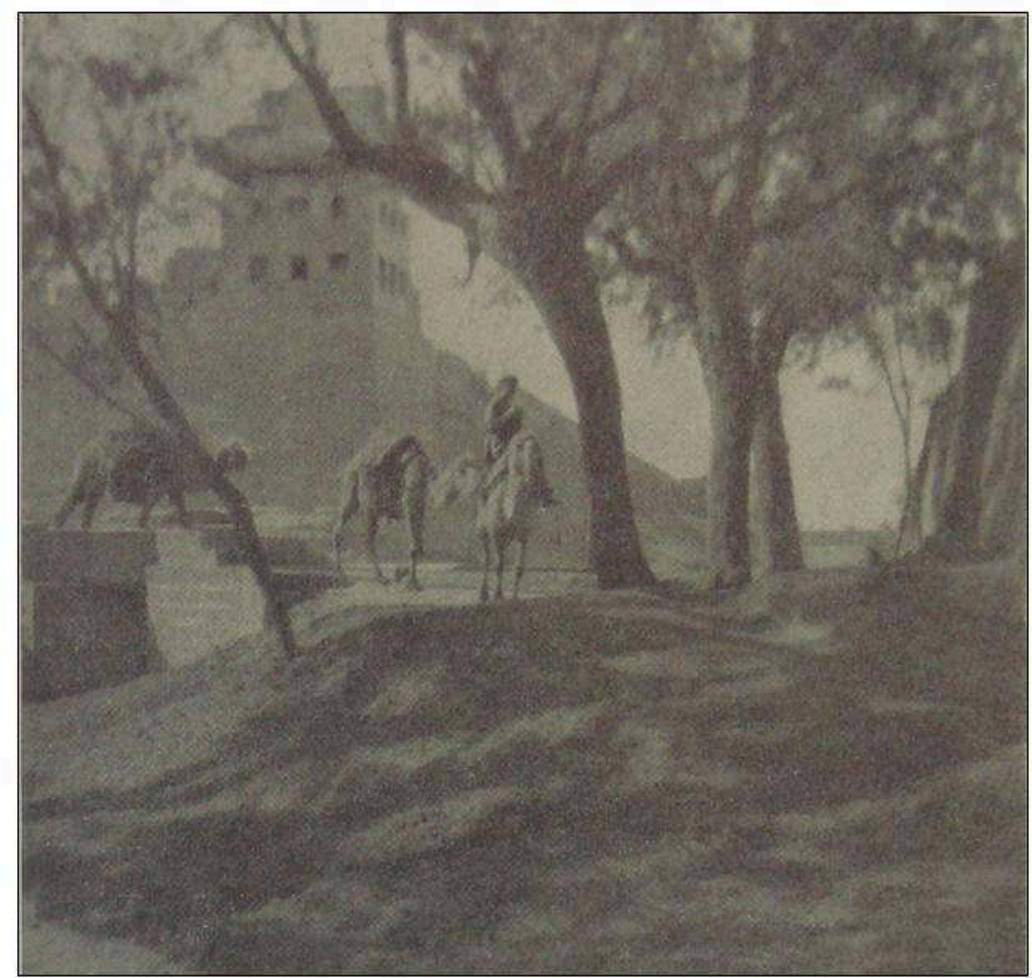

Kawamura Torakichi 川村虎吉, Rakuda no iru fükei 駱駝の いる風景 (Paysage avec chameaux), Nihon shashin nenkan, 1929, p. 27. 


\section{Figure 16}

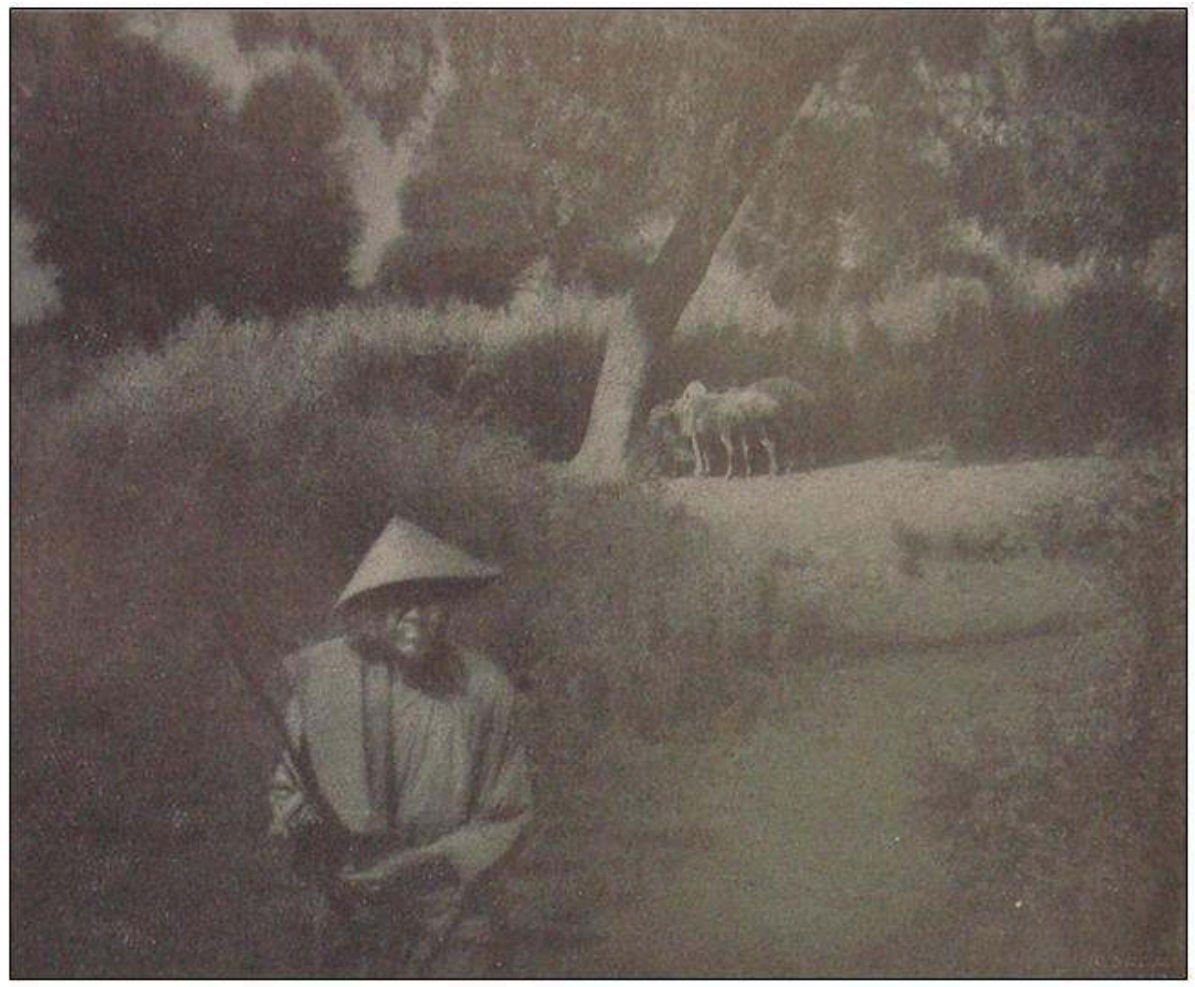

Shiba Yōson 志波陽村, Jōka no fūkei 盛夏の風景 (Paysage d'été), Nihon shashin nenkan, 1929, p. 37.

\section{Figure 17}

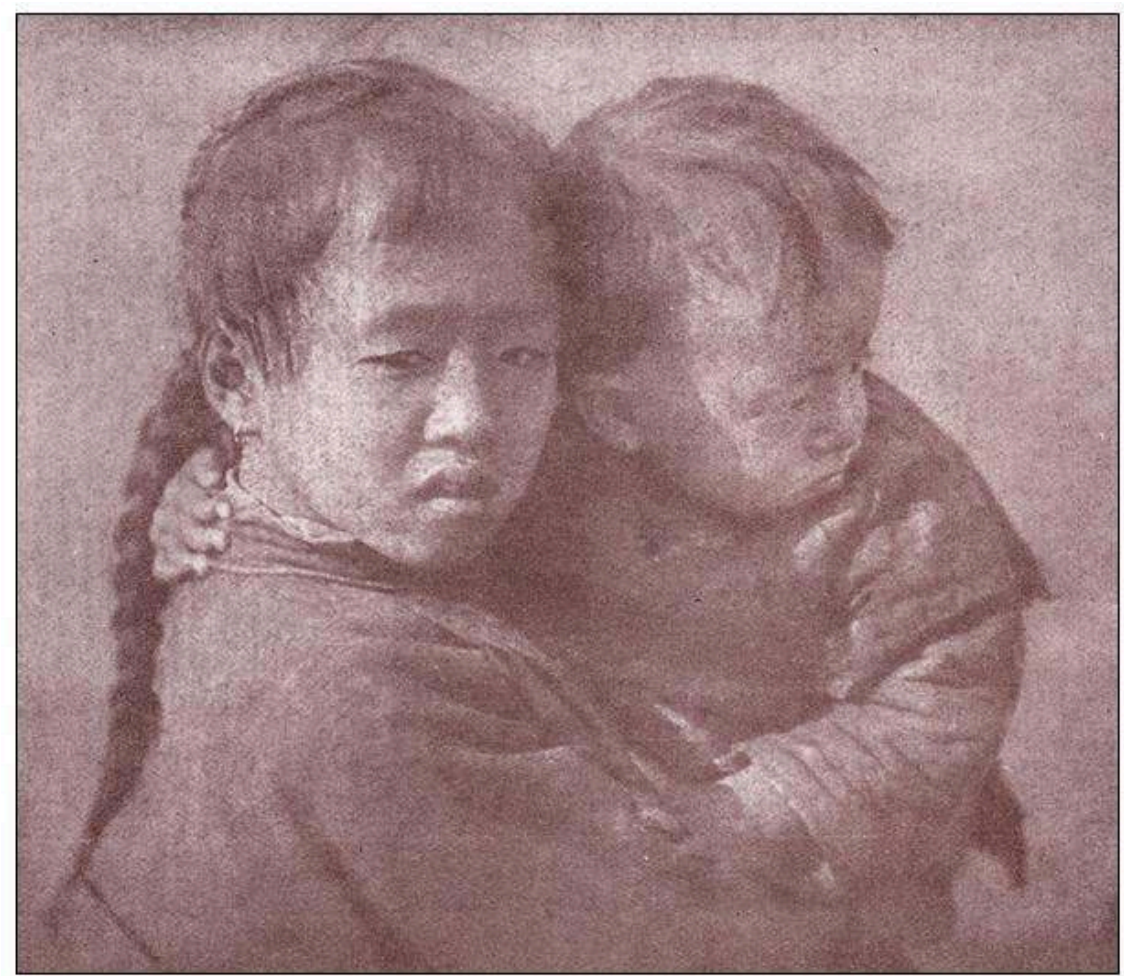

Yamaguchi Yoshiharu 山口義治, Dōjo no zō 童女の像 (Portrait de fillettes), Nihon shashin nenkan, 1930, p. 31. 
Figure 18

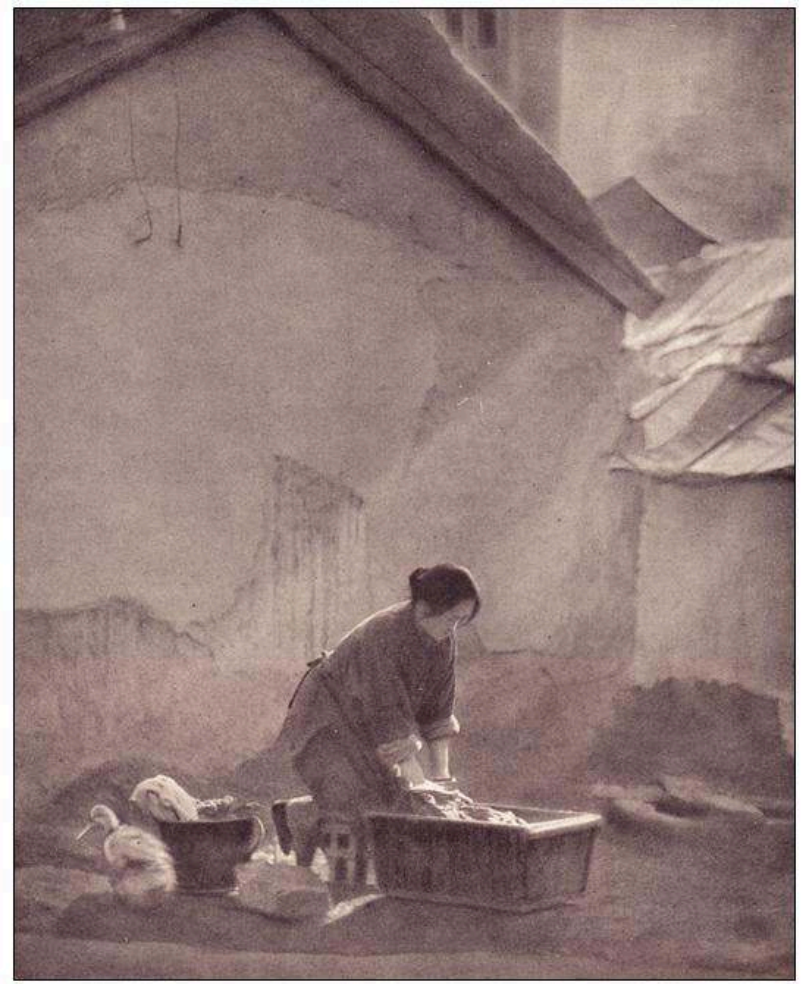

Tsuchida Kan.ichi 土田寛一Shizu no me 賎の女 (Femme de basse condition), Nihon shashin nenkan, 1931, p. 69.

21 La représentation du monde rural et d'animaux de ferme n'est pas spécifique au pictorialisme de Mandchourie. Nombreux sont les exemples traitant de ce thème dans les photographies pictorialistes réalisées sur l'archipel. C'est le traitement du sujet qui diffère, reflétant la différence du regard porté par le photographe sur la campagne de l'archipel et du continent, les représentations du monde rural japonais évoquant souvent un monde traditionnel idéalisé. De même, les représentations de paysans ou d'enfants pauvres de l'archipel existent bien à cette époque, mais sont principalement l'œuvre de photographes modernistes tel que Yasui Nakaji 安井仲治 (1903-1942), qui a réalisé de nombreux portraits de travailleurs pauvres dans une série conçue comme un reportage sur la condition sociale de ceux-ci. Les pictorialistes, quant à eux, se plaignaient de la difficulté à trouver dans les campagnes japonaises des sujets dont la pauvreté ne viendrait pas gâcher leurs œuvres ${ }^{30}$. 


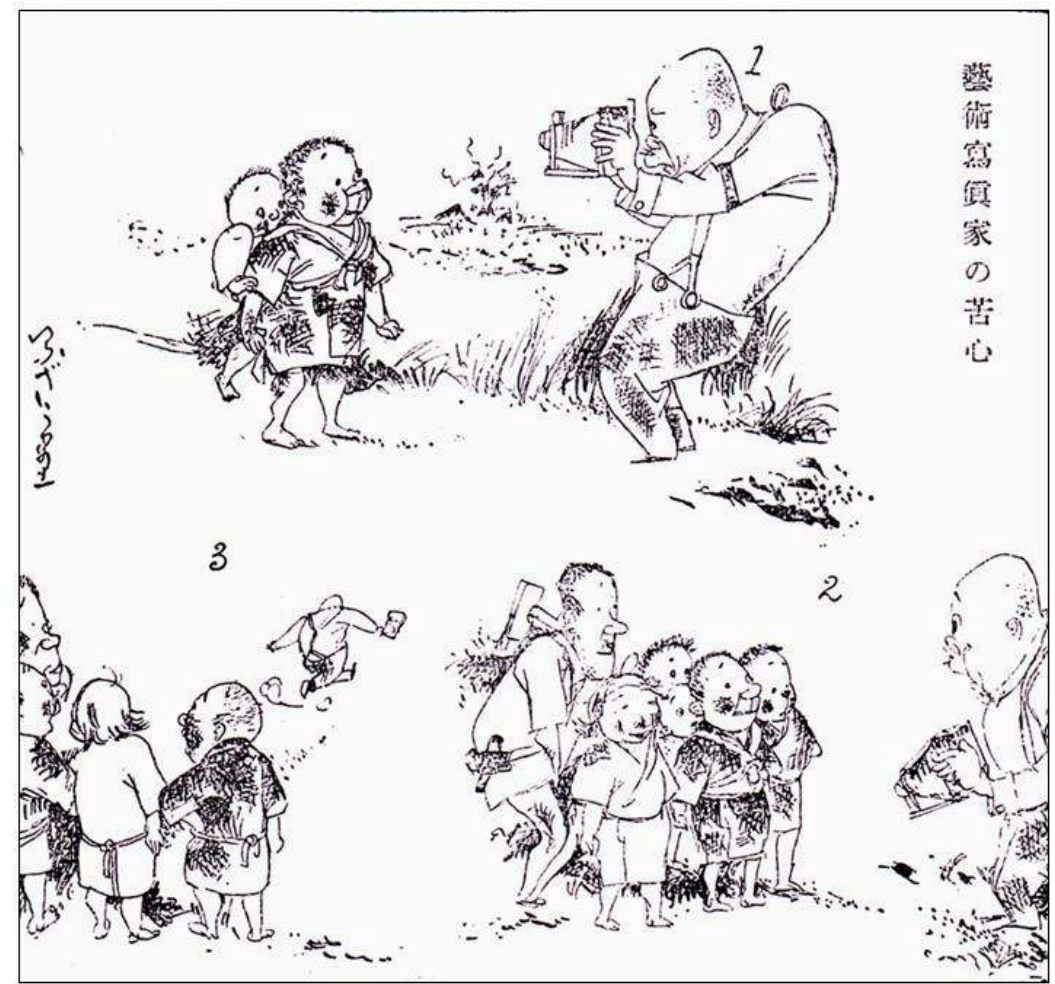

Geijutsu shashinka no kushin 芸術写真家の苦心 (Les malheurs du photographe artiste), Asahi kamera, juillet 1930, p. 111.

Ces œuvres évoquent une certaine image de la Mandchourie, telle qu'elle était perçue par une partie des Japonais à la fin des années 1920 : territoire porteur de promesses de prospérité industrielle, région exotique mais qui pouvait rappeler par certains traits la campagne japonaise, terre étrangère dont l'aventure, voire le danger, n'étaient pas absents. Ceci amène à s'interroger sur le degré d'implication des photographes pictorialistes dans le processus d'intégration des populations locales par les Japonais ce qui peut à la fois passer par le regard du photographe et par le regard porté par le spectateur de l'image -, et sur la façon dont le pictorialisme a pu servir à intégrer la Mandchourie dans la représentation que les Japonais - à tout le moins une partie d'entre eux - avaient de leur pays.

Sur le premier point, il est difficile de se prononcer : les auteurs des images citées n'ont pas expliqué leurs intentions ni les rédacteurs leurs choix - les commentaires, lorsqu'ils existent, se limitent à des données techniques - ; par ailleurs, aucune étude n'existe sur le sujet. Ce manque d'information irait plutôt dans le sens d'une non implication du monde de la photographie pictorialiste, quand il ne place pas sur un pied d'égalité les œuvres de photographes de la Mantetsu et celles plus personnelles des autres amateurs. Sur le second point, en revanche, il existe des éléments de réflexion. Si la Mandchourie avait une forte capacité à susciter dans les années 1920 déjà un certain attrait, confinant parfois au romantisme, c'est parce qu'elle était présentée aux Japonais comme une vaste terre vierge, distincte en cela de la Corée ou de Taiwan, densément peuplées ${ }^{31}$. La Mandchourie rurale était imaginée comme un espace vide et plat, comme une vaste frontière attendant l'installation des Japonais ${ }^{32}$. Ce thème est très présent dans le Manshū shashin nenkan de Fuchikami Hakuyō et illustré par la 
photographie de Terashima Manji choisie par Mariko Tamanoi ; c'est aussi ce que laissent percevoir certaines images et le sentiment de solitude ou d'éloignement qu'elles évoquent.

Certains portraits évoquent la hiérarchisation raciale et culturelle qui a en partie servi de justification à l'expansionnisme japonais, dans lequel les Chinois étaient considérés comme un peuple inférieur, caractérisé par sa saleté33. À une sorte d'angoisse exprimée par ces photographies se superpose un dédain perceptible encore aujourd'hui : la pauvreté, la saleté, l'anonymat des personnes représentées rejoignent l'analyse donnée par Louise Young des représentations scéniques de l'incident de Mandchourie dans lesquelles l'ennemi chinois était généralement une menace rôdant, sans visage ${ }^{34}$, description qui s'applique parfaitement à la photographie de Shiba par exemple. D'après Young, la vague de mépris à l'encontre des Chinois a été particulièrement forte entre 1931 et 1933 ; dans le domaine de la photographie pictorialiste, on constate que ce phénomène est un peu antérieur, beaucoup des photographies pictorialistes dépréciatrices ayant été publiées en 1929-1930. Ces portraits rejoignent également certains des témoignages recueillis par Mariko Tamanoi auprès d'anciens migrants en Mandchourie qui dressent un portrait dépréciateur de ces populations et n'incitent guère à la nostalgie ou au romantisme.

\section{Le pictorialisme, outil de matérialisation de l'utopie?}

Le pictorialisme japonais est un genre encore peu connu et étudié des historiens de la photographie; évoquer la possible implication de cette pratique artistique amateur dans l'histoire du Japon de l'entre-deux-guerres est loin d'aller de soi. Nous espérons néanmoins avoir révélé une partie de la complexité insoupçonnée qui entoure le pictorialisme dès lors que l'on se penche sur sa relation à la société japonaise. L'exemple de Mariko Tamanoi - dont les sentiments vis-à-vis de l'œuvre de Terashima Manji ont probablement été partagés par de nombreux lecteurs - rappelle que l'image photographique, loin d'être innocente, est pour partie le fruit d'une époque et d'une culture. Bien sûr, nous ne prétendons pas que le pictorialisme a été l'unique ou le plus important vecteur des représentations de la Mandchourie, mais ces images ont touché une population variée, depuis les photographes amateurs japonais jusqu'aux visiteurs des expositions internationales organisées à l'étranger dans les années 1930 - et, pardelà les décennies, ceux qui les étudient aujourd'hui.

Selon l'historienne Louise Young, "[l]'idée du Mandchoukouo n'est pas apparue brutalement, entière, mais elle a évolué au travers d'un processus d'invention et de réinvention culturelles. Autrement dit, les Japonais ont imaginé encore et encore le "Mandchoukouo" [...] ${ }^{35}$. Au vu des images diffusées dans les revues de photographie de la fin des années 1920, comment ne pas concevoir que le pictorialisme, dans un genre et selon des objectifs différents de la photographie commerciale, n'ait pas lui aussi joué un rôle dans ce processus ? La communication institutionnelle en constituait parfois la fin - commandes de la Mantetsu -, parfois l'origine - reflet d'idées acquises par les photographes à titre personnel et traduisant en image des conceptions courantes dans les années 1920 et 1930. De nombreuses photographies pictorialistes montrent un territoire hostile; d'autres évoquent la puissance de l'industrie japonaise et la possibilité d'un retour à un mode de vie agricole traditionnel et prospère. Ce sont ces dernières qui semblent susciter aujourd'hui encore de la nostalgie chez certains 
spectateurs - alors que ce genre de représentations poétiques de la Corée ou de Taiwan est rare dans la photographie pictorialiste.

Une des caractéristiques du pictorialisme est d'associer la valeur indicielle de la photographie (en d'autres termes, le fait qu'une photographie renvoie à une chose réelle) à une forme de représentation onirique et atemporelle; c'est qui en a fait un outil efficace pour matérialiser l'utopie mandchoue promue à l'époque par le gouvernement japonais. Néanmoins, certains photographes ont su faire du pictorialisme un moyen de transmettre leur perception de la Mandchourie dans des images d'un genre différent de celles de ce que nous avons appelé le "pictorialisme engagé »; il ne faut pas y voir alors une forme d'engagement, mais plutôt l'expression d'une sensibilité artistique. Ces deux usages du pictorialisme montrent bien que les photographes le pratiquant ne se refusaient pas à observer leurs contemporains et qu'il est nécessaire, comme y appelait d'ailleurs Fuchikami en 1930, de reconnaître le rôle joué par le pictorialisme dans la société japonaise de l'entre-deux-guerres.

\section{NOTES}

1. Tamanoi Mariko, Memory Maps: The State and Manchuria in Postwar Japan, University of Hawai'i Press, 2009, 211 p., p. 2.

2. Le pictorialisme est aujourd'hui désigné par le terme pikutoriarizumu ピクトリアリズム. Kaigashugi shashin 絵画主義写真 et kaigateki shashin 絵画的写真 (photographies picturales) étaient les expressions employées alors, la seconde étant la plus courante dans les années 1920 et 1930. Dans certaines histoires de la photographie japonaise (voir par exemple Nishimura Tomohiro 西村智弘, Nihon geijutsu shashin-shi 日本芸術写真史 — 浮世絵からデジカメまで (Histoire de la photographie artistique au Japon), Bigaku shuppan 美学出版, 2008, 477 p.), on trouve également l'expression pikutoriaru shashin ピクトリアル写真 (traduction littérale de l'anglais pictorial photography), dont aucune occurrence n'a pu être trouvée dans les documents d'époque consultés.

3. Sur la diversité des pictorialismes européens, on pourra se reporter au catalogue de l'exposition La Photographie pictorialiste en Europe 1888-1918, organisée au musée des Beaux-arts de Rennes d'octobre 2005 à janvier 2006 : Michel Ribemont et Patrick Daum (dir.), La Photographie pictorialiste en Europe 1888-1918, Le Point du Jour Éditeur/Musée des Beaux-arts de Rennes, Cherbourg/Rennes, 2005, $343 \mathrm{p}$.

4. Thierry Gervais et Gaëlle Morel, La Photographie : histoire-techniques-art-presse, Larousse, coll. «Comprendre et reconnaître ", 2008, 239 p., p. 171.

5. Comme toute périodisation, celle touchant au pictorialisme japonais connaît des variantes, liées aux critères retenus pour caractériser le genre.

6. Parmi les photographes pictorialistes occidentaux les plus connus, on peut citer les Britanniques Julia Margaret Cameron (1815-1879) et Peter Henry Emerson (1856-1936), les Américains Edward Steinchen (1879-1973) (fig. 2) et Alfred Stieglitz (1864-1946) (fig. 3), les Français Robert Demachy (1859-1936) (fig. 4) et Charles E.J.Constant Puyo (1857-1933), l'Allemand Heinrich Kühn (1866-1944).

Pour une présentation plus détaillée du pictorialisme occidental, se reporter à Thierry Gervais et 
Gaëlle Morel, La Photographie: histoire-techniques-art-presse, op. cit., p. 150-165 ainsi que Mary Warner Marien, Photography: A Cultural History, $2^{\mathrm{e}}$ édition, Laurence King Publishing, 2006, 544 p., pp. 171-200.

7. Nippon, lancé en octobre 1934 par le photographe et designer Natori Yōnosuke 名取洋之助 (1910-1962), était un trimestriel destiné à promouvoir la culture japonaise auprès du public d'Europe de l'Ouest, et était de ce fait publié en quatre éditions différentes (anglaise, française, allemande et espagnole). Sa publication s'acheva avec le numéro de septembre 1944. Front (1942-1945) était un illustré édité en quinze langues différentes dont la publication était placée sous le contrôle de l'armée. Ces deux périodiques de propagande ont bénéficié de la participation de photographes de renom tels que Domon Ken 土門拳 (1909-1990) pour le premier et Kimura Ihee 木村伊兵衛 (1901-1974) pour le second et du travail des graphistes Natori pour Nippon et Hara Hiromu 原弘 (1903-1986) pour Front.

8. Voir à ce sujet l'article de Gennifer Weisenfeld, «Publicité et propagande : le modernisme comme méthode ", in Jean-Jacques Tschudin et Claude Hamon (dir.), La Société japonaise devant la montée du militarisme - Culture populaire et contrôle social dans les années 1930, Philippe Picquier, 2007, 238 p., p. 47-70, consacré au rôle de la photographie moderniste dans le développement de la photographie publicitaire et de la propagande politique dans les années 1930 et 1940.

9. Louise Young, Japan's Total Empire: Manchuria and the Culture of Wartime Imperialism, University of California Press, 1998, 487 p., pp. 259-260.

10. À la suite de sa victoire sur la Russie en 1905 , le Japon se vit accorder par le traité de Portsmouth la concession des droits d'exploitation relatifs au chemin de fer. Ce fut le début d'une politique d'expansion fondée sur l'exploitation minière et supportée par la Compagnie sudmandchourienne de chemin de fer (Minami Manshū tetsudō kabushiki gaisha 南満州鉄道株式会社), ou Mantetsu 満鉄, compagnie semi-privée créée en 1906. Le 18 septembre 1931, une voie du Mantetsu fut l'objet d'un acte de sabotage imputé aux Chinois (incident de Mandchourie ou Manshū jihen 満州事变), ce qui fournit aux troupes japonaises le prétexte pour occuper la zone. Ceci déboucha, en mars 1932, sur la proclamation de l'État du Mandchoukouo, à la tête duquel les Japonais placèrent le « dernier empereur » chinois, Pu Yi.

11. Cette revue, publiée à partir de 1925 par la compagnie Asahi shinbun, proposait à ses lecteurs un annuaire des clubs de photographes amateurs, des magasins de matériel photographique et des studios photographiques sur tout le territoire japonais, colonies comprises. Ce recensement se faisait sur la base de la déclaration volontaire; ces nombres constituent des données a minima.

12. Moukden est le nom mandchou de la ville. Son nom japonais est Hōten 奉天, et son nom chinois Fèngtiān 奉天.

13. Tōkyō-to shashin bijutsukan 東京都写真美術館監 (dir.), Nihon no shashinka : kindai shashinshi wo irodotta hito to denki-sakuhinshû mokuroku 日本の写真家 : 近代写真史を彩った人と伝記・作 品集目録 (Les Photographes japonais qui ont marqué l'histoire de la photographie moderne biographies, œuvres), Nichigai Associates 日外アソシエッツ, 2005, 483 p., p. 188.

14. Le Manshū shashin nenkan porte également le titre anglais de Manchuria Photographic Annual. Cet annuaire a été conçu selon la maquette de la revue Hakuyō et proposait soixante-dix-huit photographies accompagnées des explications de l'auteur et des commentaires de Fuchikami. Manshū shashin nenkan était publié par la maison d'édition japonaise Chūnichi bunka kyōkai (Association culturelle sino-japonaise), spécialisée dans la publication d'ouvrages sur la Mandchourie.

15. Fuchikami Hakuyō 淵上白陽 (dir.), Manshū shashin nenkan-Manchuria Photographic Annual, 満 州写真年鑑 Manchuria Photographic Annual, Dairen Nitchū bunka kyōkai 大連日中文化協会, 1930, $78 \mathrm{p}$.

16. Publiée officiellement par la Mantetsu de septembre 1933 à janvier 1944, cette revue était dédiée à la promotion de la politique japonaise au Mandchoukouo au travers des œuvres des membres de l'Association des photographes de Mandchourie. Voir Anne W. Tucker, Dana Friis- 
Hansen, Kaneko Ryūichi (dir.), The History of Japanese Photography, Museum of Fine Arts, Houston, 2003, 405 p., p. 381.

17. Akiyama Tetsusuke est une des figures principales du monde de la photographie japonaise des trois premières décennies du vingtième siècle, et en particulier de la photographie amateur et du pictorialisme: entré chez le fabricant-importateur de matériel photographique Konishi Roku 小西六 (actuel Konica) en 1903, fondateur d'un des plus importants clubs de photographes amateurs, la Société d'études photographiques de Tōkyō (Tōkyō shashin kenkyūkai 東京写真研 究会), en 1907, il enseigna à l'école de photographie Konishi et fut membre-fondateur de la Fédération photographique du Kantō (Zen Kantō shashin renmei 全関東写真聯盟) en février 1926.

18. Dentiste de profession, proche de Fuchikami Hakuyō, il se fit connaître dans les années 1920 par la publication de photographies pictorialistes, reconnaissables à leurs déformations optiques, dans des revues de photographie amateur.

19. Originaire de Nagoya, membre du club de photographes amateurs le plus important du Kansai, le Aiyū shashin kurabu 愛友写真俱楽部 à partir de 1919, il entra à la Mantetsu en 1929 à l'invitation de Fuchikami et se spécialisa dans la photographie de paysages.

20. Tamanoi Mariko, Memory Maps: The State and Manchuria..., op. cit., p. 1.

21. Soit, en 1929, 4,62 \% des œuvres publiées ; en 1930, 8,25 \% ; en 1931, 4,54 \% ; en 1933, 5,68 \%.

22. Cet annuaire, situé en fin de volume, dénombre les clubs de photographes amateurs et leurs membres, les photographes professionnels et les magasins de matériel photographique, département par département. Une limite importante dans l'usage de ces chiffres est que le recensement repose sur l'autodéclaration.

23. Ceci peut s'expliquer par le fait que l'historiographie de la photographie s'est principalement intéressée aux œuvres de photographes pictorialistes célèbres - dont ceux au service de la Mantetsu -, au détriment de la myriade de photographes amateurs dont l'œuvre et la biographie sont peu connues, mais qui constituent pourtant la grande majorité des pictorialistes.

24. Gregory J. Kasza, The State and the Mass Media in Japan 1918-1945, University of California Press, 1993 (1988), 335 p., p. 34.

25. Le corpus des œuvres réalisées sur l'archipel par des photographes pictorialistes et publiées dans les revues photographiques dans les années 1920 montre l'apparition d'une esthétique urbaine, minoritaire dans le style pictorialiste mais qui devint un des thèmes majeurs de la Nouvelle Photographie dans les années 1930. En revanche, la représentation d'un monde rural japonais encore ancré dans des modes de vie traditionnels était un thème récurrent du pictorialisme japonais. Voir Sandrine Dalban-Tabard, L'Affirmation du monde de la photographie dans le Japon des années 1920, Thèse de doctorat, Inalco, 2010, 361+55 p., p. 191-200.

26. Basé à Moukden.

27. Il existe bien quelques rares exemples de représentation pictorialiste d'animaux de ferme sur l'archipel, mais sur un mode différent (fig. 11).

28. Ville de la province du Liaoning, alors partie de la Mandchourie.

29. L'âne était un des symboles évoquant la Mandchourie dans le Japon des années 1930.

30. Un dessin humoristique intitulé Geijutsu shashinka no kushin 芸術写真家の苦心 (Les malheurs du photographe artiste) paru dans le numéro d'Asahi kamera de juillet 1930 illustre cela. Il montre un photographe en sortie à la campagne qui est sur le point de photographier un enfant crasseux portant son frère ou sa sœur sur le dos. Ils sont alors rejoints par un groupe d'enfants et un adulte. Le photographe s'enfuit devant cette situation probablement trop réaliste à son goût (fig. 19).

31. Tamanoi Mariko, Memory Maps: The State and Manchuria in Postwar Japan, op. cit., p. 2. Cette allégation était connue des observateurs occidentaux des années 1930 et réfutée par certains d'entre eux. Voir Lee Hoon K., "Korean Migrants in Manchuria”, Geographical Review, vol. 22, no. 2, 
avril 1932, pp. 196-204 ; Owen Lattimore, "Chinese Colonization in Manchuria", Geographical Review, vol. 22 no. 2, avril 1932, pp. 177-195.

32. Louise Young, Japan's Total Empire: Manchuria and the Culture of Wartime Imperialism, op. cit., p. 15.

33. Pierre-François Souyri, «La colonisation japonaise: un colonialisme moderne mais non occidental », in Marc Ferro (dir.), Le Livre noir du colonialisme, Robert Laffont, coll. « Hachette Littératures ", 2003, 566 p., p. 543-574, p. 566 ; Louise Young, Japan's Total Empire: Manchuria and the Culture of Wartime Imperialism, op. cit., p. 95.

34. Ibid., p. 15.

35. Ibid., p. 18.

\section{RÉSUMÉS}

Si la présence japonaise en Corée, en Mandchourie ou encore à Taiwan s'est appuyée sur des discours la décrivant et la justifiant, elle s'est également accompagnée d'une iconographie vectrice de stéréotypes les corroborant visuellement.

Cet article interroge les représentations de la Mandchourie et le rôle joué par les photographes japonais dans la création et la diffusion de ces représentations dans les années 1930. Pour ce faire, il s'appuie sur l'étude d'œuvres pictorialistes, en d'autres termes sur des photographies à visée artistique, mode de représentation alors pratiqué majoritairement par des amateurs.

Nous montrerons ainsi dans quelle mesure ce type de photographies, par la vision de la Mandchourie proposée par le photographe et la réception qui en fut faite par le spectateur, a pu constituer un des outils de matérialisation de l'utopie mandchoue alors diffusée au Japon et interrogerons le degré d'implication de ces photographes dans la propagande officielle.

\section{INDEX}

Index géographique : Mandchourie

Mots-clés : Fuchikami Hakuyō (1889-1960), photographie, pictorialisme, Sakakibara Shōichi, Terashima Manji (1898-1983)

Thèmes : histoire de l'art

Keywords : Fuchikami Hakuyō (1889-1960), Sakakibara Shōichi, Terashima Manji (1898-1983), Pictorialism, Photography, Art History, Shōwa period

Index chronologique : Shōwa (1923-1945)

\section{AUTEUR}

\section{SANDRINE DALBAN-TABARD}

Centre d'études japonaises, Inalco 